\title{
The functional design method for buildings (FDM) with gamification of information models and AI help to design safer buildings
}

\author{
JUKKA SELIN \\ Department of Information Technology \\ South-Eastern Finland University of \\ Applied Sciences Ltd \\ Patteristonkatu 2, 50100 Mikkeli FINLAND \\ jukka.selin@xamk.fi www.xamk.fi
}

\begin{abstract}
We have developed a method that enables better taking into account of need of space of actions of moving objects in a building when Building Information Modeling (BIM) is used. The 3D spatial objects for real space requirements of actions created with our method can be used with various design tools as such in dimensioning or to generate video game colliders defining the need of space. Together with the gamified model of the structure from BIM they enable dimensioning in designs and simulations. We can dimension spatial objects for example related to safety. There are a lot of needs and applications for our methods. It is possible to design buildings and other structures that fit their purpose well.
\end{abstract}

\section{INTRODUCTION}

$\mathrm{I}_{\mathrm{n}}^{\mathrm{n}=\mathrm{s}}$ this article we present the utilization of the Functional Design Method (FDM) "Value Add Data / VAddD" together with the Gamified BIM Information Model during the simulation of emergency evacuation from a supermarket. The simulation relates to the joint R\&D and piloting effort of the S-E Finland University of Applied Sciences (XAMK) and our industrial construction partners. The Functional Design Method is based on the Finnish patent by our senior lecturer and Licentiate of Science Jukka-Pekka Selin. The patent was derived from an earlier internal innovation report [1], [2], [3].

The core idea in the FDM is to capture the space requirements of real action in three dimensions and then create a new 3D spatial object. The new 3D objects represent the maximum spatial space requirements of actions. When using Construction CAD these objects can be used in dimensioning different spaces. The goal can be both to fit an action to a space or alternatively ensure that an object can reach all the needed places in a space. If the Information Model in the form of an IFC (Industrial Foundation Classes) file is gamified it becomes possible to use the spatial objects to realise e.g. adaptation capable game colliders around game objects. In that case we can test the fitting of different actions or reaching capabilities of objects to stationary parts of the construction. We can also perform simulations.

In our piloting program we used a real construction project of our industrial partner, a Finnish supermarket [4]. In piloting we simulated the emergency evacuation from a supermarket with a gamified model by utilising AI (Artificial Intelligence) based navigation of moving avatars in the model of the market. In the simulation we had avatars with individual behavioristic profiles and sets of rules. We made
MARKKU ROSSI

RTD and Services

South-Eastern Finland University of Applied Sciences Ltd

Patteristonkatu 2, 50100 Mikkeli FINLAND markku.rossi@xamk.fi www.xamk.fi

the avatars evacuate themselves using an AI engine and individual parameters along evacuation routes that were rapidly chosen after a fire alarm.

\section{METHODOLOGIES AND SOFTWARE USED IN THE RESEARCH}

The maximum space requirement in dimensions $\mathrm{x}, \mathrm{y}$ and $\mathrm{z}$ can be derived from video clips of real human actions. In this method the real actions are videoed with at least two video cameras that are situated at right angles. The need of space from the actions in three dimensions is measured and a corresponding geometrical object is created. This $3 \mathrm{D}$ object in IFC file format is compatible with different CAD software. The goal is to ensure that the required actions can fit the space under design [1], [2], [3].

During the research program the application that is BIM compatible and is meant for Lifecycle Management of Building Data, and the application extension Value Add Data of Xamk R\&D, were used for creating 3D objects. The application extension realizes the functionality of the FDM. The Colliders describing the space requirements for different actions to the gamified model were created via the execution of the Value Add Data software [5].

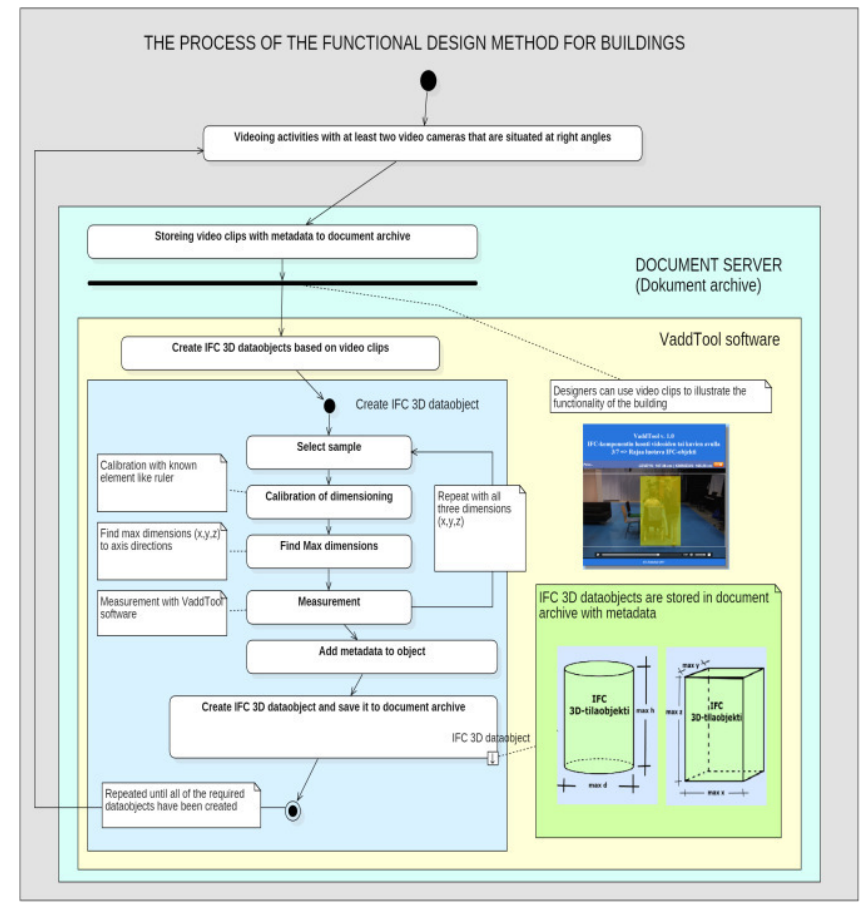

Fig 1. The process of the FDM for Buildings [5]. 
The Information Model used with the supermarket consists of several submodels that have been created by the designers of architecture, structures and different subsystems, with different CAD tools. As an example, the architecture model has been designed with the ArchiCAD software by Graphisoft SE. All submodels of the supermarket have been received for piloting in the IFC format [4], [6]. The submodels have been converted by the tools of the Open Source software family IfcConvert to the OBJ format [7]. The files that were in the OBJ format have been further optimized and shortened with the Open Source software MeshLab.

The metadata that are in the IFC files have been transferred by using the XML format. The conversion from IFC into XML has been performed with the IfcConvert. The gamification itself has been performed with the Unity game engine. When simulating the emergency evacuation we used the Navigation extension of the Unity engine. This extension brings possibilities to use AI based navigation schemes [8].

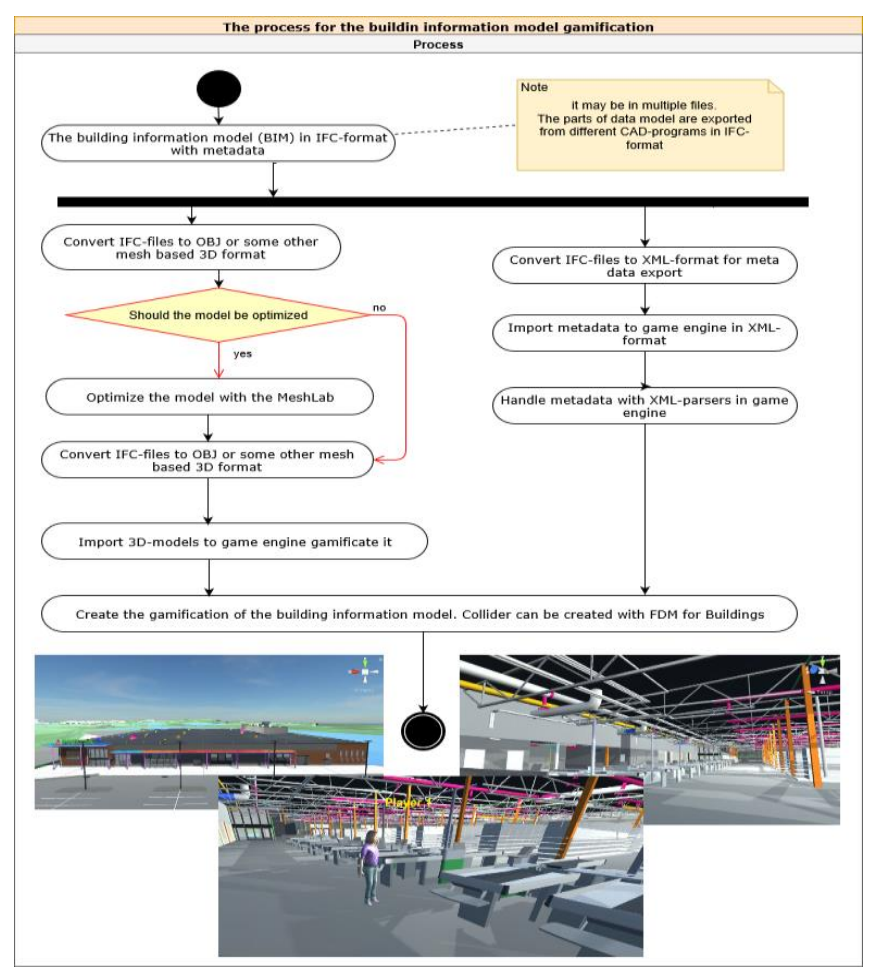

Fig 2. Our process for gamifying Information Models.

The schematic above describes the process developed by us to gamify Information Models of buildings. The basic principle of the process is that the gamification should be possible independent of the choice of the original CAD design tool. The only requirement is that the $\mathrm{CAD}$ tool supports the IFC format and can export both data and metadata into an IFC file according to the BIM recommendations [6].

Into a model gamified this way we can bring spatial objects generated by our FDM. These VAddD spatial objects can also support designs as such or if further converted into Game Colliders.

\section{THE FUNCTIONAL DESIGN METHOD FOR BUILDINGS TOGETHER WITH GAMIFICATION OF INFORMATION MODELS HELP TO DESIGN SAFER BUILDINGS}

We created pilots to study different possibilities and applications for the FDM. We utilized an Information Model of a real supermarket. Our partner, the construction company U.Lipsanen $\mathrm{Oy}$ is currently building the supermarket. We received the Information Model as several IFC submodels, so we were able to test our procedure to gamify models represented according to the recommendation IFC (Fig 2) so that also the metadata can be input to the gamified model [4].

The main goal was to be able to use open software. For that reason we ended up to use the IfcConvert software from the IfcOpenShell library to convert IFC files into mesh models supported by game engines. Among the formats of mesh model formats we selected the OBJ format. We had a need to optimize and radically reduce the amount of polygons approximating the surfaces, especially related to pipes. To do this we chose the open MeshLab software that has support for the OBJ format. We also used the IfcConvert to create XML files from IFC and to bring the metadata of the models together with the 3D model into the game engine [6], [7].

With the aid of the gamified Information Model we piloted the usage of FDM in the emergency evacuation with an AI navigation control used in a Unity game environment [8]. We placed profiled or individually parametrized avatars randomly inside the supermarket and then commanded the avatars to proceed to the closest emergency exit with the aid of the AI engine. Around each avatar we placed game colliders that described the space needed for the avatar when mobile, according to the avatar profile. Such avatar can use different aids to overcome limited mobility when moving. An avatar using an aid like a rollator usually needs more space than an average person. Each of the avatars also possessed an individual moving speed.

With FDM we generated the 3D spatial objects representing spatial needs for actions. The description of the cases for avatars can be seen in the following table (Table 1).

TABLE I.

MAXIMUM SPACE REQUIREMENTS FOR THE DIMENSIONING OF THE COLLIDERS CREATED BY THE FDM

\begin{tabular}{|l|l|}
\hline \multicolumn{1}{|c|}{ The dimensioned Action } & Space Requirement (m) \\
\hline A walking person & $0.50 \times 1.90 \times 0.70(\mathrm{x}, \mathrm{y}, \mathrm{z})$ \\
\hline A running person & $0.65 \times 1.90 \times 0.90(\mathrm{x}, \mathrm{y}, \mathrm{z})$ \\
\hline $\begin{array}{l}\text { The assistant walks with the wheelchair } \\
\text { user on behind the wheelchair }\end{array}$ & $1.30 \times 1.90 \times 1.00(\mathrm{x}, \mathrm{y}, \mathrm{z})$ \\
\hline The wheelchair user without the assistant & $1.00 \times 1.50 \times 1.00(\mathrm{x}, \mathrm{y}, \mathrm{z})$ \\
\hline The user with rollator & $0.90 \times 1.90 \times 0.75(\mathrm{x}, \mathrm{y}, \mathrm{z})$ \\
\hline The user with crutches & $0.90 \times 1.90 \times 1.00(\mathrm{x}, \mathrm{y}, \mathrm{z})$ \\
\hline
\end{tabular}

From each of the cases we generated an avatar prototype that has colliders corresponding to the table. In this pilot the characteristics of the colliders were specified on a relatively common level. We felt that this accuracy is adequate from 
the piloting of simulation method point of view. The avatar profiles could well be much more individual and more different types of avatars representing different customers of the supermarket could be created.

For accurate and realistic simulations corresponding to real situations it is necessary to increase both the number of variables and to increase the number of movement functions that have a partially random outcome. The number of simulation drives should be high when we want to apply statistical methods to the outcomes of the individual simulation drives. The results of the statistical studies would show how well the supermarket supports safety and show hints how to further increase safety by design. Different building designs could be simulated and compared. After iterating with different designs it is possible to reach the functionality requirements.

In our piloting we were mainly interested in the feasibility of the FDM in this kind of dimensioning and simulations. We used in our pilots some randomness in the collisions in between different objects to increase realism. The piloting was performed by using the Unity game engine and the C\# programming language. We added a simulation extension to the gamified Information Model of the supermarket. The simulation extension creates a predefined number of avatars with different characteristic profiles. In the beginning of the simulation the avatars are located at their initial locations around the floor area, derived from random values of parameters. The characteristics for different types of avatars are in the table above (Table I).

In the simulation we used the Navigation tools of Unity, based on AI. With the tool we could create a navigation map situated on the floor level of the supermarket. More generally, the map could include any stationary objects of the gaming world. With the aid of the navigation map an avatar can now be commanded to progress to the needed target area so that the AI tool steers the movement of the avatar. The events during the navigation phase can be controlled by numerous different navigation parameters. Among the features that can be controlled are the maximum physical difficulty level of obstacles that can be over- or sidewise passed, and the intensity of effort in movement.

We realized the simulation in a way that the customer avatars with spatial needs according to their profiles are moving and directed by the AI towards emergency exits. The situation can be monitored by virtual cameras situated in the gamified Information Model. Each of the avatars are also carrying their own cameras that can be switched on when necessary.

The development of the situation can now be monitored from all angles. With the simulation application developed You can quickly control e.g. the number of customers, randomness of events and navigation parameters. It is also possible to change and define quickly the target-objects that are the destinations of the avatars. The avatar game objects that represent supermarket customers and are used in the simulation can in principle look like whatever the artist decides, but we increased the realism of the simulation by creating 3D avatars that look like typical shoppers. We also made them move according to kinetic data from an earlier human motion capturing session with cameras. Their movement looks therefor quite natural.

The realism of the simulation can be enhanced by adding different effects. Here we wanted to simulate a fire in a supermarket and we added a smoke effect to the gamified model. The progress of the fire follows a well known Fire Intensity Curve that is a generalized model about how the fires progress (Fire Intensity against elapsed time) [9]. The following picture (Fig 3) visualizes a generalized Fire Intensity curve that represents the intensity of the fire in majority of cases. In addition, the picture visualizes the phases of the evacuation and parameters used in the case of a supermarket.

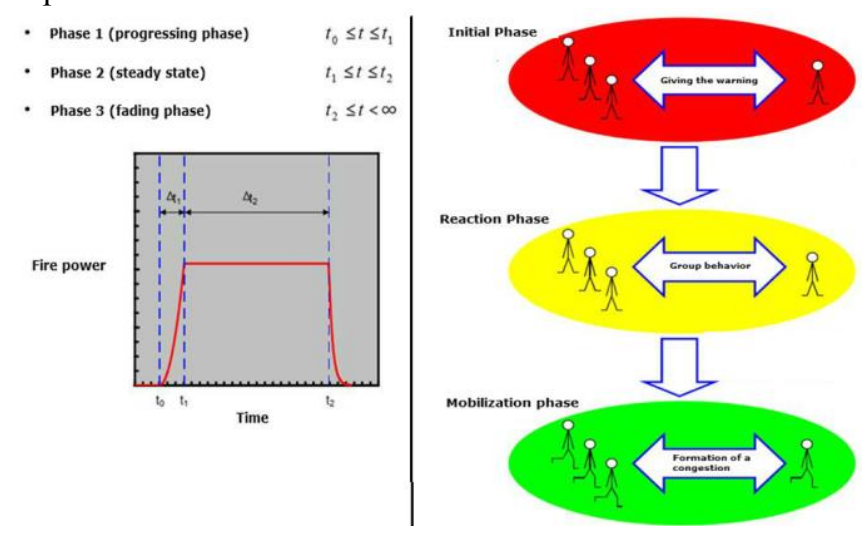

Fig 3. On the left side a generalized Fire Intensity curve that represents the intensity of the fire in majority of cases. On the right side the phases of an emergency evacuation [9].

The fire develops inside a building usually so that the environment becomes intolerable for humans. The cause can be different poisonous and irritating gases and the heat. Because of the fatal environment one should remove humans from the building as quickly as possible [9].

At the University of Lund the research aimed to find out how long it takes before the environment close to the fire becomes intolerable for humans. Also the VTT Technical Research Centre of Finland Ltd has performed research on the topic. In both research programs the critical factor was found to be the smoke occupying the whole volume of the building. The scattering of the research results is however relatively high [9], [10].

Based on the before mentioned research results we set the critical elapsed time after the start of the fire to 25 minutes with a standard deviation of 8 minutes. We put 500 customers into the supermarket corresponding to the daily rush hour. Our simulation can however adapt easily to a wide range of parameters for each case. The evacuation of humans from the buildings can be divided into three main phases. The phases are called the preliminary phase, the reaction phase and the mobilization phase. The total time elapsing in the evacuation follows the formula $\mathrm{t}=\mathrm{t}$ (initial) $+\mathrm{t}($ reaction $)+$ 
$\mathrm{t}$ (mobilization). The durations of these times are naturally very individual, so we need to include random variables related to the behaviour of people to our calculations. We realized the simulation application of an emergency evacuation by utilizing scientific studies mentioned above. We set the parameters of the dangerous situation (here a fire) so that the situation can be divided into the above mentioned phases. It was possible to freely change the durations of the phases. We also designed the customer (avatar) profiles so that there is randomness in the profiles and the process follows the phases of an evacuation [9].

The following picture (Fig 4) shows a view from the simulation application on the display during the run of a process performed by the Unity game engine.

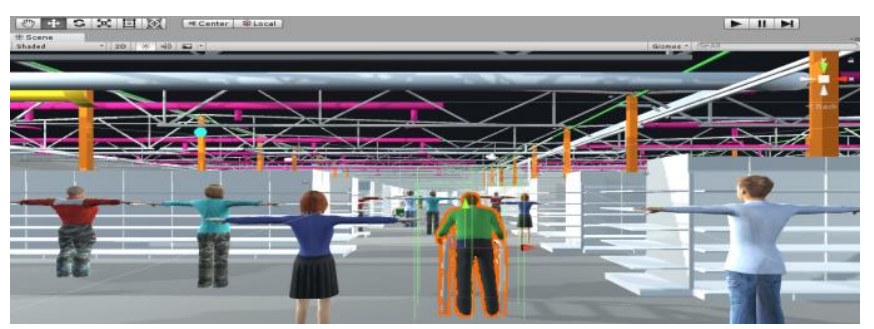

Fig 4. A simulation application realized with the Unity game engine. A game collider corresponding to the spatial need of an avatar is visible on the picture.

The event that causes the need for evacuation can be parametrized and the seriousness and location can be varied. We can test a situation where a fire restricts access to a certain area and can even prevent using certain emergency exit.

After the simulation starts the game objects corresponding to profiled supermarket customers begin to move towards the selected emergency exits. The following picture (Fig 5) visualizes a situation where the fire simulation is active and the profiled customers are trying to move towards the emergency exits. Their spatial needs and profile settings affect their moving speed and route when they navigate past shelves and other structures, while interacting with the other customers. We can observe how the customers occasionally prevent each other using the most direct routes.

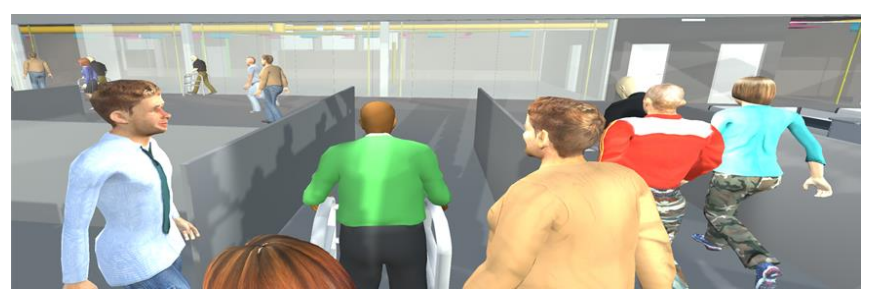

Fig 5. The simulation is active and smoke begins to enter the interior of the supermarket. The profiled customers (game objects) are rushing in chaos towards the defined emergency exits under the control of the AI engine (the reaction phase and the mobilization phase).

The structure of the simulation application is such that it saves the movements of the profiled customers to a log file. The applications also recorded for every customer the moment when he moved from a phase to the next. We also recorded to the $\log$ of the collisions with the other avatars and the moment of time when the customer was able to exit the building. Then the avatar reached the emergency exit that was the target location of the navigation.

The simulation was built so that the profiled customers possessed randomness according to the range found out in the studies [9], [10] and [11] during the initial and reaction phases. The simulation run specific log file generated gave a lot of valuable data about the functioning of the building in an emergency. With the aid of the logs it is e.g. possible to study the success level of different safety increasing measures. The $\log$ includes data for e.g. finding out when $90 \%$ of the customers have left the building or how much a new exit reduces the time of evacuation and also what the best place for a new exit is. In the exemplary simulation presented here all of the 500 customers could exit the building in 15 minutes. Over $90 \%$ of the customers had exited already in less than 10 minutes from the beginning of the fire. The last ones to exit were the individuals who have long reaction times and who are using aids for reduced mobility. When we made the distance between the shelf units narrower the evacuation times increased significantly especially with high numbers of people. Correspondingly, extra exits shortened the evacuation times, as expected. According to this simulation the supermarket was found to be safe enough in fire situations with the designed types of corridors and exits.

We also tested during the simulations a situation where the cameras are videoing from the point of view of the customers. We gave each customer an own camera enabling us to switch to the view from any of these cameras. It was especially interesting to note how an aged customer using mobility supporting aids and moving slowly suffers from collisions with other customers moving move swiftly. The following picture visualizes the emergency evacuation event from the point of view of e.g. an older customer moving slowly and using a rollator. This fact could also be found from the generated log files. The following picture (Fig 6) visualizes the emergency evacuation event from the point of view of e.g. an elderly.

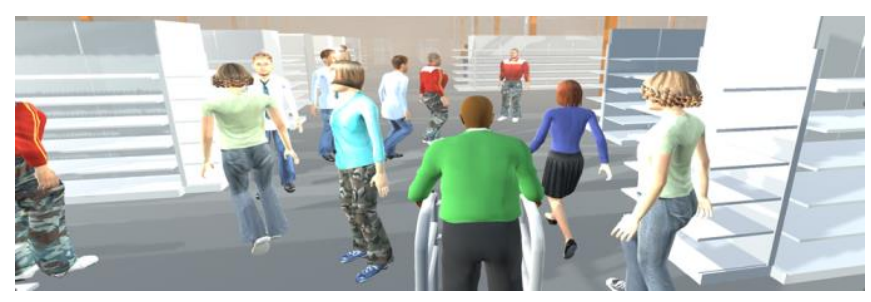

Fig 6. Simulation showing the point of view of a slowly moving elderly using a rollator.

Finally we simulated a situation where the viewer is a TPCtype object (Third Person Controller) who tries to exit the building. Also in this case we have a set role and spatial needs that were dimensioned by using the FDM. We conclude that this could be an excellent way to practice doing the emergency evacuation. By using the multiplayer functions of Unity we could simulate situations where several players are practicing emergency evacuation or generally any co- 
operation with other players. The following picture (Fig 7) visualizes a situation where each of the players of the simulation is moving a profiled avatar that has settable parameters. The players can do different roles like rescue or leading the evacuation operation. In the picture the player has entered the supermarket and encounters there customers who are rushing out. This way we could practice beforehand the actions during emergencies from the point of view of different actor roles.

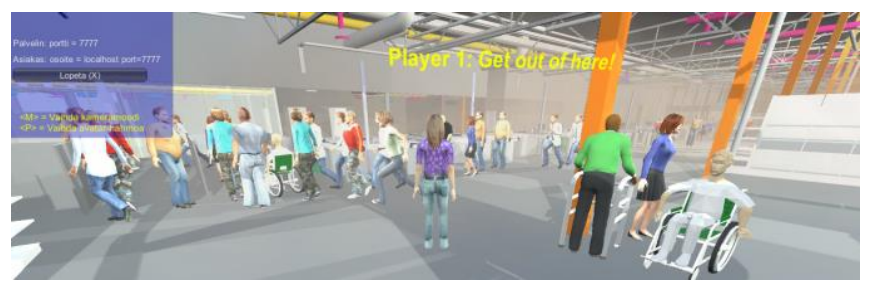

Fig 7. A TPC player (Player1) has entered the supermarket after having received an alarm, performs rescue and assistance work and encounters customers who are rushing in panic.

The approach of this pilot appeared to be very interesting and relevant. The FDM brought clear added value to the piloting, because the different individual spatial needs could be taken into account in the simulation. According to our industrial partner the principle to combine the simulation of emergency evacuation with the usage of the FDM is a very good idea. We are planning to further develop these methods in our current research project.

The insertion of multiplayer functionality and co-operation of a community into this kind of simulation was especially rewarding. When the Information Model is gamified and we crowdsource the emergency evacuation simulation we could get valuable knowledge and ideas from a large group of people to enhance the accessibility and safety of a supermarket. We plan to develop these methods and tools further in the future.

\section{RESULTS AND CONCLUSIONS}

The pilots presented strengthened our view that the Functional Design Method VAddD developed earlier is a useful and practical tool for designing buildings and that it is flexible to cover different purposes and application environments. The method suits also to different design challenges of infrastructure and built environment in addition to building design. It helps to perform the designs based on end users' activities in the buildings. The method is also independent on what $\mathrm{CAD}$ ecosystem was used to design the first 3D model. The pilot presented belong to our research program for the construction and building industry.

We conducted a small scale interview among our R\&D partners related to the research results. We interviewed the industrial contact persons who are either managers or designers from the construction industry. One of the designers interviewed was specialized on gaming.

As a summary from the results of the interviews the usage of BIM, the gamification of Information Models and the
Lifecycle Management of the Information Model were seen very welcome and needed development paths for the construction industry. The interviewed also said that the FDM is a very good idea for taking the human actions into account in all kinds of design challenges better than before. All new methods belonging to this category of methods were seen as welcome development. The partners thought that the simulation of the emergency evacuation scene was successful and stated that the FDM brings clear added value and new feasibility because You can now profile the users of the building and create individual characteristics and spatial needs to the avatars used. This way the simulation can be made very realistic and the results really bring new data about the building design and its functionality.

The partners of our R\&D program think that the FDM for buildings and the pilots utilizing the methods are interesting, bring added value to the design processes and so are definitely worth further studies. Exactly to this direction should the design of buildings according to their opinion be developed in the future. When this pilot analysis cases are combined with further analysis of other actions in the building we have a large number of analysis results from a complete toolset of analysis fulfilling the needs in structural design, simulation, building automation design, training and even lifetime measurement and control during the whole lifecycle. Our vision is to adapt and expand the methods to develop virtual user interfaces or digital twins to buildings for the whole lifetime, beginning from the design phase.

\section{REFERENCES}

[1] An action space defining object for computer aided design. Finnish Patent 125913, granted on April 15, 2016. Fourteen claims. Current owner : Xamk. Inventor : Selin, Jukka-Pekka. Is the origin of the PCT patent application WO 2014/154942 A1.

[2] An action space defining object for Computer Aided Design. PCT patent application WO 2014/154942 A1. Applicant Mikkeli University of Applied Sciences Ltd (Jukka-Pekka Selin).

[3] Rossi, Markku J. and Bhargav, Dave, Digitalization and quality enhancement initiatives in sw assisted design processes in building and construction industries. 9th International Conference on Computer Engineering and Applications, Dubai, 2015. ISBN 978-1-61804-276-7.

[4] Construction Company U.Lipsanen Oy, A Building Information Model of Commercial Center in IFC format, Pieksämäki, Finland, 2018.

[5] Selin Jukka., Rossi Markku J., Simulation of universal design by a functional design method and by Gamification of Building Information Modeling. FedCSIS, 2016 Federated Conference, Gdansk, Poland, 2016. ISBN 178-8-3608-1090-3 and 978-1-5090-0046-3, IEEE.

[6] BuildingSMART International. The official website of the IFC-format (ISO 16739:2013). Accessed on April 18, 2018. http://www.buildingsmart-tech.org/specifications/ifc-releaces

[7] The official website of the IfcOpenShell-project. Accessed on April 18, 2018. http://ifcopenshell.org/

[8] Unity Inc. The official website of the Unity game engine. Accessed on April 18, 2018. https://www.unity3d.com/

[9] Paloposki Tuomas, Myllymäki Jukka, Weckman Henry. VTT Oy. Julkaisu. http://www.vtt.fi. (ISBN:951-38-6114-7). Accessed on May 11, 2018.

[10] Magnusson, S.-E., Frantzich, H. \& Harada, K. Fire safety design based on calculations - Uncertainty analysis and safety verification. Lund, SE: Lund University, 1995. 120 s. (Report 3078.) ISSN 1102-8246k.

[11] Weckman, H. Rakennuksista poistumisen laskennallinen arviointi. VTT Oy, Julkaisu. ISBN 951-38-5133-8, ISSN 1235-060 